\title{
A Prognostics and Health Management for Information and Electronics-Rich Systems
}

\author{
Michael PECHT*
}

電子情報機器における故障予知と健全性管理

マイケル・ペクト

* CALCE Electronics Products and Systems Center, University of the Maryland, and City University of Hong Kong PHM Centre

\begin{abstract}
概要 Prognostics and systems health management (PHM) は，システムにおける設計・製造・環境および使用条件・維持の 複雑さに起因して顕在化しつつある信頼性の諸問題を解決するための技術掞よび方法論に関する分野の 1 つである。過去 10 年 間, 情報機器・電子機器を対象にして故障の早期警告, 計画的な維持管理, システムの品質向上, 寿命延伸および市場での不 良における no-fault-found の原因の 1 つである断続的な故障の検出などを可能とする手法としてPHMの研究が行われてきた。本 稿では, 電子情報機器を対象としたPHMの現状について述べる。PHMのアプローチとしては, 故障モデルベースとモニタリ

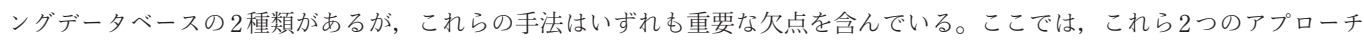
を融合させた新しい手法(Fusion approach)について紹介する。Fusion approachの故障予知への実装の例として, プリント回路板 に適用した事例を紹介する。
\end{abstract}

\section{Introduction}

Extremely high operational availability of information systems has been historically difficult to achieve because of the lack of understanding of the actual application environments and operating conditions, and their effects on system degradation and failure. Furthermore, the fact that most failures in information systems are intermittent makes many predictive methods unacceptable. ${ }^{1)}$ Combining this complexity with the $40-85 \%$ no-fault-found (NFF) failure rate seen in system failure analysis suggests that current reliability practices need improvement. In particular, traditional approaches to failure mitigation have failed because of the reliance on averaged accumulated historical field data (e.g., Mil-Hdbk-217,2) Telcordia SR-332 (formerly Bellcore), ${ }^{3)}$ and CNET/RDF (FIDES) (European) ${ }^{4), 5)}$ ), rather than relying on in situ data from a particular system. In fact, studies ${ }^{6), 7)}$ have reported that these methods are inaccurate and misleading (i.e., they provide inconsistent results for any given system subject to given conditions). This is a major reason why the US Army has abandoned these approaches. In addition, the IEEE notes that information system failures are in some sense inevitable, because the current methods of assessing information systems have fundamental flaws. ${ }^{8)}$

Another and significantly more scientific approach to reliability assessment and prediction falls under the general heading of physics-of-failure (PoF). ${ }^{9-12)}$ The PoF approach involves a number of steps, which generally include some form of failure modes, mechanisms and effects analysis (FMMEA), feature extraction, and remaining useful life (RUL) estimation. ${ }^{9)}$ To implement this approach, the potential failure modes, mechanisms, and sites of the system based on the lifecycle loading conditions must be identified. The stress at each failure site is obtained as a function of loading conditions, geometry and material properties of the system. Damage models are then used to determine fault progression and RUL. The failure models require input, such as material properties, geometry, and environmental and operating loads.

PoF-based prognostic methodologies have been applied to estimate RUL in electronic assemblies and components such as power supply chips on a DC/DC voltage converter printed circuit board (PCB) assembly ${ }^{13)}$; PCBs subjected to loads under an automobile hood ${ }^{14), 15)}$; electronics subjected to thermo-mechanical loads ${ }^{16)}$; and for monitoring, recording, and analyzing the life cycle vibration loads for estimation of the RUL of PCBs using cumulative damage laws. ${ }^{17)} \mathrm{A}$ PoF-based tool has been developed for real time prediction of RUL of PCBs exposed to thermal cycling environments. ${ }^{18)}$ The tool integrates information from sensors, PoF models, and data fusion algorithms to en- 
able prognostics. ${ }^{\dagger}$

Using the models developed, it is possible to calculate the damage accumulation and RUL for known failure mechanisms. This is one of the advantages of model-based approaches. As the model-based approaches take into account degradation caused by environmental conditions such as thermal loads, humidity, vibrations, and shock, they can be used to estimate damage in situations where systems are in a non-operating state such as during storage and transportation. Knowledge of failure mechanisms, along with the monitored loads and system parameter data may allow for identification of the nature and extent of the fault. For example, power cycling of insulated gate bipolar transistor (IGBT) modules leads to wire-bond and die attach fatigue that causes a change in the collector-emitter voltage. The magnitude of change in the voltage is an indicator of the extent of the degradation in the component. ${ }^{19), 20)}$

Development of the models requires detailed knowledge of the underlying physical processes that lead to system failure, ${ }^{21)}$ and in complex systems, it is often difficult to create dynamic models representing the multiple physical processes occurring in the system. ${ }^{22}$ Although JAPAN ARTICLE has shown that this is possible, it remains one of the limitations of model-based approaches. A requirement of the PoF model-based approach is that system-specific knowledge, such as geometry and material composition, is necessary which may not always be available. Further, failure models or graph-based models are not suitable for detection of intermittent system behavior as they are modeled for specific degradation mechanisms or for the diagnosis of specific faults respectively. Sudden changes in system parameters that characterize intermittent faults are not accounted for in these models.

Consequently, there is a pressing need to develop new technologies and methods that utilize in situ system operational and environmental conditions to detect performance degradation and faults, and avoid and manage system failures. Furthermore, these new technologies and methods must account for soft faults and intermittent failures, ${ }^{\dagger}$ which are some of the most common failure modes in today's information systems. $^{23)}$

The most promising discipline of technologies and methods with the potential of solving reliability, availability, and maintainability problems is called prog- nostics and systems health management (PHM). PHM of information and electronics-rich systems is a means of providing advance warnings of failure, enabling forecasted maintenance, improving system qualification, extending system life, and diagnosing intermittent failures, which can lead to field failure returns exhibiting no-fault-found symptoms.

The purpose of this paper is to present an assessment of the state of practice in PHM of information and electronics-rich systems and some of the R\&D opportunities and challenges. We discuss an R\&D approach to PHM that fuses two of the current approaches, model-based and data-driven approaches, to overcome their individual limitations while retaining the advantages of both. Then we present an example of implementation of the fusion approach to electronics-rich systems.

\section{State of Practice in PHM}

Traditionally, PHM has been implemented using approaches that are either model-based or data-driven. The model-based approaches takes into account the physical processes and interactions (the physics of failure) of the components in the system. ${ }^{21)}$ The datadriven approaches use statistical learning and machine-learning algorithms to detect changes in data and identify the deviation from normal or healthy conditions, thereby enabling diagnostic and prognostic measures to be calculated. ${ }^{1)}$ Fusion prognostic methodologies combine the strengths of the model-based and data-driven approaches.

In the system modeling approach, mathematical functions or mappings, such as differential equations, are used to represent the system. Statistical estimation techniques based on residuals and parity relations (the difference between the model predictions and system observations) are then used to detect, isolate and predict degradation. ${ }^{21), 22)}$ Estimation techniques such as Kalman filters, particle filters, and parity relations are commonly used to calculate the residuals. For example, this approach to prognostics was demonstrated for lithium ion batteries ${ }^{24)}$ where a lumped parameter model was used along with extended Kalman filter and particle filter algorithms to estimate remaining useful life (RUL). Model-based prognostics methods are currently being developed for power semiconductors, ${ }^{19), 25)}$ digital electronics components and systems such as microprocessors in avionics, ${ }^{26)}$ switched-mode 
power supplies, ${ }^{27)}$ and diagnostics of software health. ${ }^{28)}$

The PoF approach utilizes knowledge of a system's life-cycle loading conditions, geometry, and material properties to identify potential failure mechanisms and estimate RUL. ${ }^{1)}$ This approach is based on the understanding that failures occur due to fundamental mechanical, chemical, electrical, thermal, and radiation processes.9) However, as previously noted, there are challenges related to this approach based on the information need to create these models.

Data-driven techniques are used to learn from the data and intelligently provide valuable decision-making information. They are based on statistical learning and machine learning algorithms applied to data collected in situ to determine the state of health of a system. Deviation from the normal or healthy states is considered as an anomaly, which also enables fault isolation. Furthermore, the trends in the data and the deviation from the healthy states are used to estimate the time to failure of the system.

In this approach, in situ monitoring of environmental and operational loads and system parameters is carried out. The data collected is analyzed using a variety of techniques depending on the type of data available. For example, if data representing the healthy and faulty states of the system are available, a supervised learning approach is used. When data for only one class, such as the healthy state of the system, are available, then the semi-supervised approach is used. A third approach is the unsupervised learning approach, which is used when no labeled data are available. Decisions about the system health are typically made using assumptions regarding the system data. It should be noted that employing both the supervised and semi-supervised learning techniques requires reliable training data. This is important, as the classification of incoming data is dependent on the training data, and unreliable training data will lead to errors in detection. Furthermore, data is usually present in two forms: continuous (also known as parametric) which are real-valued data from sensors (such as temperature, humidity, vibration, etc.), and categorical (also known as non parametric) can be error or fault messages that are either of textual nature or binary flags. Some of the fault messages can be triggered when the subsystem behaves outside preset operating parameters. Data driven methods can combine algorithms an- alyzing both types of data for better and more accurate estimates.

Prognostics based on data-driven methods use past history to infer the future, continually update the prediction of RUL, and provide an estimate of the associated prediction uncertainty. Data-driven methods are very useful for complex systems where the knowledge of the underlying physics are absent and the health of systems is to be assessed. They can be used as blackbox models as they learn the behavior of the system based on monitored data and hence do not require system-specific knowledge. Further, data-driven approaches can be applied to complex multivariate systems, such as computer servers and notebooks where a large number of parameters are monitored. This is because data-driven approaches can be used to model the correlation between parameters and interactions between subsystems as well as effects of environmental parameters using in situ data from the system. Furthermore, these methods can identify the parameters that are indicative of the health of the system instead of using all variables, hence reducing dimensionality and making the performance of the algorithm better. Pattern recognition and statistical techniques employed in detecting changes in system behavior have been shown to be suitable for diagnostic purposes. This attribute makes it possible to detect sudden changes in system parameters allowing for detection and analysis of intermittent faults. This information can be used along with trending or other techniques measuring the deviation (such as Takagi-Sugeno) to estimate the remaining useful life. For example, Markov state models used features extracted from notebook computers to predict state transition probabilities and times. ${ }^{29)}$ Symbolic time series analysis and Mahalanobis distance were used for feature extraction by Kumar and Pecht. ${ }^{29)}$ Pattern recognition algorithms and statistical techniques for early fault detection have also been developed for computer servers. ${ }^{30), 31)}$ Trending methods to predict the RUL of electronics have been suggested using continuously monitored data. ${ }^{33)}$ Other applications in electronics where data-driven approaches have been used for RUL estimation include global positioning systems ${ }^{32)}$; avionics ${ }^{33)}$; power electronics devices (IGBTs) used in avionics ${ }^{34)}$; and aircraft electrical power systems. ${ }^{35), 36)}$

One of the limitations of data-driven approaches lies in the requirement of training data. Data-driven ap- 
proaches depend on historical (e.g., training) system data to determine correlations, establish patterns, and evaluate data trends leading to failure. In many cases, there will be insufficient historical or operational data to obtain health estimates and determine trend thresholds for failure prognostics. This is true for example in stored, standby, and non-operating systems, which are nevertheless subject to environmental stress conditions, and in systems where failures are infrequent. A solution to this problem is to incorporate (or fuse) system models, such as (PoF) models, with the datadriven models.

\section{A Fusion Prognostics Approach}

Fusion prognostic methodologies combine the strengths of the model-based and data-driven approaches, in order to estimate RUL under both operating and non-operating life cycle conditions, detect anomalous behavior or intermittent faults, identify precursors to failure for effective maintenance planning, and identify the potential processes causing system failure and the nature and extent of the fault for effective maintenance strategies.

The first step in the process is to identify parameters that can be monitored in situ to aid in determining the real time state of health of the system. Environmental and system parameter data are required to be monitored for the diagnosis and prognosis of a system's health in real time. The process of identifying the parameters for monitoring can be aided by an understanding of the physical processes that lead to system failure. FMMEA, virtual simulations, information from maintenance records and qualification tests, or expert knowledge can be used for identifying parameters and for determining the relevant models for estimation of RUL. Understanding the physical processes occurring in the system helps in identifying critical components, possible failure sites, failure mechanisms, and their effects on the system. Appropriate sensing technology is then selected for the monitoring of the chosen parameters. The sensor data are analyzed in real time in order to assess the current state of the system and determine its RUL using information from data-driven techniques and physics-based models.

Assessment of a system's health is carried out in real time using the in situ data and anomaly detection techniques. Knowledge of the physical processes in the system can help in choosing the appropriate data- driven techniques for diagnosis and prognosis. At this same time, the nature of data will also influence the algorithm to choose, based on whether the data is continuous or categorical, high frequency, and the normality of data. One of the ways to implement anomaly detection is the application of a machine-learning approach, in which the monitored data are compared in real time against a healthy baseline to check for anomalies. This is the semi-supervised learning approach wherein data representing all the possible healthy states of the system are assumed to be available $a$ priori.

The healthy baseline consists of a collection of parameter data that represent all the possible variations of the healthy operating states of a system. The baseline data is collected during various combinations of operating states and loading conditions when the system is known to be functioning normally. The baseline can also consist of threshold values based on specifications and standards. It is important that the baseline data should not contain any operational anomalies. The presence of anomalies in the baseline affects the definition of healthy system behavior and hence causes the misclassification of data. Misclassification leads to problems such as false indications of anomalies (false alarms) or failure to detect anomalous behavior of the system (missed alarms).

Although healthy baseline data sets are important for machine learning approaches to detection, other statistical and probabilistic approaches that rely on parametric and distributional assumptions can also be used. In the machine-learning context, for example, distance-based similarity measures and other features can be extracted from multidimensional data. In addition projections and filtering of the data can also be used to extract features from the data. Detection in the machine-learning approach is largely based on these features. These techniques are particularly useful when no a priori data is available to create a baseline of healthy states.

After the anomaly detection step, the parameters that contribute significantly to the anomaly are isolated. It is important to pinpoint which parameters reflect or cause changes in system performance: they are critical in identifying and detecting system failure. Parameter isolation can be carried out using a variety of techniques, such as principal components analysis (PCA), least squares (LS) estimation, expectation maxi- 
mization (EM), and maximum likelihood estimation (MLE). Parameter isolation can also be used before anomaly detection: if training data exists, feature selection can be performed in order to identify the parameters that are only indicative of the health of the system, and the machine learning algorithms will be based on only a select-number or isolated parameters. Based on the information from the parameter isolation step, the critical parameters are used to select appropriate models from the database. The parameter isolation step helps determine the models most relevant to the type of failure or degradation the system is undergoing. Physics-based models, which use the isolated parameters as the primary inputs, are selected in this step.

Physics-based models are used to calculate the RUL of the system based on the environmental and parameter data along with information such as material properties and system specifications. Knowledge from failure mechanisms and models is also used to extract information such as failure thresholds for the measured system parameters, failure modes, stages of degradation, and labels of healthy and unhealthy conditions. Failure definitions can also be obtained by referring to other sources, such as standards and established failure criteria for the system. This input of failure definitions and labeling of healthy and unhealthy states from the model-based approach is critical in the selection of appropriate data-driven prediction methodologies for estimation of RUL. For example, a Markov model of the failure or degradation process of a system depends on modeling the transition of the system in and out of various "states". These states can, for example, be used to model the various failure mechanisms or violations of failure thresholds as defined by the model-based approach. In other words, the modelbased approach can identify precursors to failure that can be used for early annunciation and prediction of system failure.

Using the failure thresholds, methods such as time series analysis or particle filtering techniques can be applied to predict the critical parameter values over time. The time until the parameter crosses the failure threshold is estimated as the time to failure of the system. Therefore, an estimate of the RUL for the system based on the combination of information from anomaly detection, parameter isolation, physics-based models, and data-driven techniques can be calculated.

Alarms can be set off to warn the system operator of impending failure based on the value of the RUL reported. This can provide adequate time for repair or replacement of the system depending on the criticality of the application.

\section{Application of Fusion Prognostics to Electron- ics-Rich Systems}

The fusion prognostics approach was implemented on an electronics system consisting of a printed circuit card assembly subjected to temperature cycling conditions. The assembly consisted of ball grid array (BGA) packages, quad flat packages and surface mount resistors that can be found in circuit cards of electronics systems such as computers, avionics systems and most other information systems. A FMMEA analysis determined the critical modes and mechanisms affecting the assembly to be interconnect fatigue due to thermal cycling resulting in open circuit. Temperature and resistance parameters were considered parameters to detect system failure for the given loading conditions.

Anomaly detection was carried out using a datadriven residual analysis technique. The required training data (baseline) to model the healthy states of the system was created using ten cycles of in situ data. The training data was assumed to represent the healthy operating states of the BGA components. Using five out of the ten cycles from the training data, a regression model was created to capture the variation of resistance with temperature and estimate component resistance. The differences between the regression model estimates and the observations of resistance were used to obtain the residual signal. The residuals were statistically tested using a sequential probability ratio test (SPRT) algorithm to detect anomalies. ${ }^{36)}$ The remaining five cycles of training data were used in the regression model to calculate healthy residuals to train SPRT. Following this, every test observation was input into the model for estimation and then analyzed statistically for anomalies using SPRT. The SPRT alarms were set off when the mean of the residuals of the resistance shifts to a value equal to or greater than the threshold value.

Parameters causing or contributing to an anomaly can be identified by assessing the variables against physics based models from a database. In this case study, the anomalous behavior due to an increase in resistance was identified. But the change in resistance was a result of cyclic temperature loads on the system 
leading to thermal fatigue. The damage to the components and time to failure due to the thermal cycling on the components were calculated from a modified Coffin Mason formula. The mean cycle to failure for the 256 I/O BGAs was calculated to be 1,038 cycles (2750.7 h). The estimate for $10 \%$ cycles to failure was obtained as 817 cycles using Monte-Carlo analysis. The RUL can be calculated dynamically using the PoF model by updating the temperature cycle profile as it is monitored in situ.

To calculate an estimate of RUL dynamically using data-driven techniques, a failure threshold of $300 \mathrm{X}$ for the resistance parameter was obtained from the IPCSM785 standard. ${ }^{37)}$ The cycles to failure was calculated at the 601 st cycle to be 620 cycles. The RUL was calculated based on the trend from the anomaly to the defined failure threshold. The estimates of RUL from the PoF model and the data-driven technique were then used to obtain a revised conservative RUL estimate for the component. The actual failure of the component was observed after 693 cycles.

\section{Conclusions}

The failures observed in information and electronicsrich systems such as aerospace, data servers, electrical power grids, energy generation equipment, and healthcare systems has led to increased research efforts in PHM for electronics systems. Today, model-based approaches are being developed to enable estimation of RUL for electronic systems such as batteries, digital electronics, navigation systems, switched-mode power supplies and printed circuit boards subject to a variety of environmental conditions. Similarly, data-driven approaches are also being developed for diagnostics and prognostics of enterprise servers, navigation systems, and power systems used in avionics and hybrid vehicles.

The model-based and data-driven approaches that are currently used in PHM have certain advantages and limitations. The model-based approaches take into account the physical processes and failure mechanisms that occur in systems, enabling prognosis of RUL. The damage accumulation calculation can be used for life assessment even when the system is no in operation. A limitation of this approach is that it cannot detect intermittent failures, and may not always feasible to capture all the parameters leading to the failure in fieldconditions. The data-driven approach is useful when system-specific information is not available. The strength of this approach is diagnostics. This approach is capable of detecting intermittent failures, thereby reducing no-fault-founds. The limitations of this approach are that it is difficult to determine RUL without historical data, as well as the lack of a standard way of establishing failure thresholds that can be used in RUL determination. The current focus in research, therefore, is to find a means to effectively use available systemspecific information, model-based approaches, and data-driven diagnostic and prognostic techniques which are known as fusion approaches to PHM.

A fusion approach enables effective use of information from both the model-based and data-driven approaches to achieve dynamic prognosis of RUL. Analytical techniques such as FMMEA, virtual simulations, and knowledge from maintenance records and experts can help identify the proper parameters for in situ monitoring. Understanding the physical processes and system behavior can help in choosing the datadriven techniques for diagnosis and prognosis. The fusion approach is capable of detecting operational anomalies and intermittent behavior allowing analysis of the root causes of no-fault-found errors as it uses appropriate diagnostic (data-driven) techniques. Isolation of the parameters that contribute to the anomalous behavior is carried out using data-driven techniques and system knowledge and leads to the identification of the critical failure mechanisms. Based on the identification of the critical failure mechanisms, identifying the nature and extent of faults and failure modes is possible. RUL estimations can then be calculated using the failure models for the critical failure mechanisms. System understanding can help determine failure thresholds for the parameters that can be used in data-driven techniques for RUL estimation. The fusion approach therefore provides recognition of degraded, but still functional, systems. The approach implemented in our case study on electronic assemblies shows that the fusion of the data-driven and modelbased prognostic techniques can be used to effectively detect faulty behavior, determine the critical parameters and nature of a failure and estimate the RUL of the PCB assemblies. This paper uses the conservative RUL estimate from amongst the PoF model and the data-driven technique.

Current R\&D challenges in PHM for electronics-rich systems that need to be addressed include uncertainty 
analysis and investigating techniques to fuse or combine estimates of RUL from various sources to provide single fused RUL values. It is important to understand and quantify uncertainty in predictions from PHM systems for realistic decision-making. Predictions based on distribution of parameters will be more informative in making maintenance and logistics decisions rather than using point estimates. Challenges in uncertainty analysis lie in determining and quantifying all the sources that contribute to prediction uncertainties such as measurement noise, model uncertainties, and missing or unavailable training data. Further, it is also necessary to investigate and develop models and datadriven approaches that take into account uncertainty in making predictions thereby providing estimates in the form of PDFs.

In order to address the challenge in combining estimates of RUL from model-based and data-driven approaches, it is necessary to investigate techniques that can help in information fusion. These techniques while providing a single output of RUL using predictions from the model-based and data-driven should also take into account uncertainty estimates from each approach. Some techniques that have been suggested for fusing information based on Dempster-Shafer regression, fuzzy set operations, and model-based information fusion techniques. Addressing these challenges in research will help build more robust PHM systems that can be implemented for information and electronics-rich systems.

\section{Acknowledgments}

This work was in part supported by the NASA ARMD/IVHM program under NRA project 07IVHM1-07-0042 ("Reliable Diagnostics and Prognostics for Critical Avionic Systems"), City University of Hong Kong-PHM Center, and the over 50 member CALCE Consortium at the University of Maryland.

(2010.8.24-受理)

\section{References}

1) M. Pecht: "Prognostics and Health Management of Electronics," New York (NY), Wiley-Interscience, 2008

2) United States Department of Defense: "Mil-Hdbk-217f Note 1 Reliability Prediction of Electronic Equipment," 1995

3) Telcordia Technologies: “Special Report SR-332: Reliability Prediction Procedure for Electronic Equipment," Pis- cataway, NJ, Telcordia Customer Service, Issue 1, May 2001

4) Union Technique de L'Electricité: “Recueil de données des fiabilite: RDF 2000, Modèle Universel Pour Le Calcul De La Fiabilité Prévisionnelle Des Composants, Cartes Et Equipements Electroniques (Reliability Data Handbook: RDF 2000-A Universal Model for Reliability Prediction of Electronic Components, PCBs, and Equipment)," July 2000

5) Délégation Générale pour l'Armement: "FIDES Guide 2004-Reliability Methodology for Electronic Systems," 2004

6) M. Priore, P. Goel, and R. Campbell: "TRW Automotive Assesses PRISM Methodology for Internal Use," J. Reliab. Anal. Cent., Vol. 10, No. 1, pp. 14-19, 2002 (first quarter)

7) J. G. Elerath: "Specifying Reliability in the Disk Drive Industry: No More MTBF's," Proceedings of the Reliability and Maintainability Symposium, January 2000, pp. 194-199

8) IEEE: “IEEE Standard 1413: Standard Methodology for Reliability Prediction and Assessment for Electronic Systems and Equipment," New York, NY, 1998

9) M. Pecht and J. Gu: "Physics-of-Failure-Based Prognostics for Electronic Products," Trans. of the Institute of Measurement and Control, Vol. 31, No. 3/4, pp. 309-322, 2009

10) M. Pecht and A. Dasgupta: "Physics-of-Failure: An Approach to Reliable Product Development," 1995 Int'1 Integrated Reliability Workshop Final Report, Lake Tahoe, CA, pp. 1-4, Oct. 22-25, 1995

11) C. Pusarla, A. Dasgupta, M. Pecht, and A. Christou: “A Physics-of-Failure Design Philosophy Applied to FlipChip Bonds," Microelectronics Int'1, No. 36, pp. 6-12, Jan. 1995

12) M. Pecht and A. Dasgupta: "Physics-of-Failure: An Approach to Reliable Product Development," Journal of the Institute of Environmental Sciences, pp. 30-34, Sept./ Oct. 1995; update of M. Pecht: "Physics-of-Failure: An Approach to Reliable Product Development," Proc. of Institute of Environmental Sciences, Chicago, IL, pp. 111-117, Aug. 1995; update of "Physics-of-Failure Approach to Design and Reliability Assessment of Microelectronic Packages," Proc. of the First Int'l Sym. on Microelectronic Package and PCB Technology, Beijing, China, pp. 175-180, Sept. 19-23, 1994

13) J. Simons and D. Shockey: "Prognostics Modeling of Solder Joints in Electronic Components," Proceedings of the 
2006 IEEE Aerospace Conference, March 2006

14) S. Mishra, M. Pecht, T. Smith, I. McNee, and R. Harris: "Remaining Life Prediction of Electronic Products Using Life Consumption Monitoring Approach," Proceedings of the European Microelectronic Package Interconnection Symposium, June 2002, pp. 136-142

15) A. Ramakrishnan and M. Pecht: "A Life Consumption Monitoring Methodology for Electronic Systems," IEEE Trans. Compon. Packag. Technol., Vol. 26, No. 3, pp. 625-634, 2003

16) P. Lall, M. Hande, C. Bhat, V. More, R. Vaidya, and J. Suhling: "Algorithms for Prognostication of Prior Damage and Residual Life in Lead-Free Electronics Subjected to Thermo-Mechanical Loads," 11th Intersociety Conference on Thermal and Thermomechanical Phenomena in Electronic Systems, May 2008, pp. 638-651

17) J. Gu, D. Barker, and M. Pecht: "Prognostics Implementation of Electronics under Vibration Loading," Microelectron. Reliab., Syst. Prognostics Health Manage, Vol. 47, No. 12, pp. 1849-1856, 2007

18) V. Rouet, F. Minault, G. Diancourt, and B. Foucher: "Concept of Smart Integrated Life Consumption Monitoring System for Electronics," Microelectron. Reliab., Vol. 47, No. 12, pp. 1921-1927, 2007

19) N. Patil, J. Celaya, D. Das, K. Goebel, and M. Pecht: "Precursor Parameter Identification for Insulated Gate Bipolar Transistor (IGBT) Prognostics," IEEE Trans. Reliab., Vol. 58, No. 2, pp. 271-276, 2009

20) Y. Xiong, X. Cheng, Z. J. Shen, C. Mi, H. Wu, and V. Garg: "A Prognostic and Warning System for Power Electronic Modules in Electric, Hybrid, and Fuel Cell Vehicles," IEEE Trans. Ind. Electron., Vol. 55, No. 6, pp. 2268-2276, 2008

21) J. Liu, D. Djurdjanovic, K. A. Marko, and J. Ni: “A Divide and Conquer Approach to Anomaly Detection, Localization and Diagnosis," Mech. Syst. Signal Process., Vol. 23, No. 8, pp. 2488-2499, 2009

22) G. Vachtsevanos, F. L. Lewis, M. Roemer, A. Hess, and B. $\mathrm{Wu}$ : "Intelligent Fault Diagnosis and Prognosis for Engineering Systems," 1st ed., Hoboken (NJ), John Wiley \& Sons, Inc., 2006

23) M. Pecht, M. Dube, M. Natishan, and I. Knowles: "An Evaluation of Built-in Test," IEEE Trans. Aerosp. Electron. Syst., Vol. 37, No. 1, pp. 266-272, 2001

$24)$ B. Saha, K. Goebel, S. Poll, and J. Christophersen: "Prognostics Methods for Battery Health Monitoring Using a Bayesian Framework," IEEE Trans. Instrum. Meas., Vol. 58, No. 2, pp. 291-296, 2009
25) D. L. Goodman: "Prognostic Methodology for Deep Submicron Semiconductor Failure Modes," IEEE Trans. Compon. Packag. Technol., Vol. 24, No. 1, pp. 109-111, 2001

26) P. W. Kalgren, M. Baybutt, A. Ginart, C. Minnella, M. J. Roemer, and T. Dabney: "Application of Prognostic Health Management in Digital Electronic Systems," Proceedings of the 2007 IEEE Aerospace Conference, March 2007, pp. 1-9

27) C. Kulkarni, G. Biswas, and X. Koutsoukos: “A Prognosis Case Study for Electrolytic Capacitor Degradation in DC-DC Converters," Annual Conference of the Prognostics and Health Management Society, September 2009

28) A. Dubey, N. Mahadevan, and R. Kereskenyi: "Reflex and Healing Architecture for Software Health Management," International Workshop on Software Health Management, IEEE Conference on Space Mission Challenges for Information Technology, July 2009

29) S. Kumar and M. Pecht: "Health Monitoring of Electronic Products Using Symbolic Time Series Analysis," AAAI Fall Symposium on Artificial Intelligence for Prognostics, November 2007

30) L. Lopez: "Advanced Electronic Prognostics through System Telemetry and Pattern Recognition Methods," Microelectron. Reliab., Vol. 47, No. 12, pp. 1865-1873, 2007

31) A. Urmanov: "Electronic Prognostics for Computer Servers," Proceedings of the 53rd Annual Reliability \& Maintainability Symposium (RAMS), January 2007, pp. $65-70$

32) D. W. Brown, P. W. Kalgren, C. S. Byington, and J. R. Roemer: "Electronic Prognostics-A Case Study Using Global Positioning System (GPS)," Microelectron. Reliab., Vol. 47, pp. 1874-1881, December 2007

33) C. S. Byington, P. W. Kalgren, B. K. Dunkin, and B. P. Donovan: "Advanced Diagnostic/Prognostic Reasoning and Evidence Transformation Techniques for Improved Avionics Maintenance," Proceedings of the 2004 IEEE Aerospace Conference, Vol. 5, March 2004, p. 34

34) B. Saha, J. Celaya, P. Wysocki, and K. Goebel: "Towards Prognostics for Electronics Components," Proceedings of the 2009 IEEE Aerospace Conference, March 2009, pp. $1-7$

35) K. Keller, K. Swearingen, J. Sheahan, M. Bailey, J. Dunsdon, K. W. Przytula, et al.: "Aircraft Electrical Power Systems Prognostics and Health Management," Proceedings of the 2006 IEEE Aerospace Conference, March 2006, p. 12 
36) N. Gebraeel and L. Hernandez: "Advanced Prognostics for Aircraft Electrical Power Systems," SAE Int. J. Aerosp., Vol. 1, pp. 1059-1063, April 2009

37) IPC-SM-785: "Guidelines for Accelerated Reliability Testing of Surface Mount Attachments," November 1992

\section{十用語解説}

prognostics：故障予知技術

intermittent failures : 断続的，あるいは一過性の故障 